\title{
Risk and Benefit for Targeted Therapy Agents in Pediatric Phase II Trials in Oncology: A Systematic Review with a Meta-Analysis
}

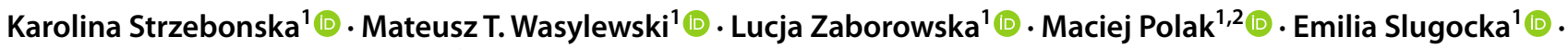

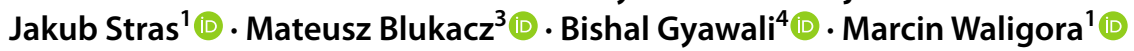

Accepted: 27 May 2021 / Published online: 10 June 2021

(c) The Author(s) 2021

\begin{abstract}
Background For research with human participants to be ethical, risk must be in a favorable balance with potential benefits. Little is known about the risk/benefit ratio for pediatric cancer phase II trials testing targeted therapies.

Objective Our aim was to conduct a systematic review of preliminary efficacy and safety profiles of phase II targeted therapy clinical trials in pediatric oncology.

Methods Our protocol was prospectively registered in PROSPERO (CRD42020146491). We searched EMBASE and PubMed for phase II pediatric cancer trials testing targeted agents. We included solid and hematological malignancy studies published between 1 January, 2015 and 27 February, 2020. We measured risk using drug-related grade 3 or higher adverse events, and benefit by response rates. When possible, data were meta-analyzed. All statistical tests were two-sided.

Results We identified 34 clinical trials (1202 patients) that met our eligibility criteria. The pooled overall response rate was 24.4\% (95\% confidence interval [CI] 14.5-34.2) and was lower in solid tumors, 6.4\% (95\% CI 3.2-9.6), compared with hematological malignancies, 55.1\% (95\% CI 35.9-74.3); $p<0.001$. The overall fatal drug-related (grade 5) adverse event rate was $1.6 \%$ (95\% CI 0.6-2.5), and the average drug-related grade 3/4 adverse event rate per person was 0.66 (95\% CI 0.55-0.78). Conclusions We provide an estimate for the risks and benefits of participation in pediatric phase II cancer trials. These data may be used as an empirical basis for informed communication about benefits and burdens in pediatric oncology research.
\end{abstract}

\section{Introduction}

Although death rates for cancers have declined by about $50 \%$ from the 1970s, cancer continues to be one of the leading causes of death in the pediatric population [1]. Globally, it is estimated that there will be 13.7 million new pediatric cancer cases by 2050 [2]. Hence, new therapeutics that

Marcin Waligora

m.waligora@uj.edu.pl

1 Research Ethics in Medicine Study Group (REMEDY), Department of Philosophy and Bioethics, Jagiellonian University Medical College, ul. Kopernika 40, 31-501 Kraków, Poland

2 Chair of Epidemiology and Population Studies, Jagiellonian University Medical College, Kraków, Poland

3 Institute of Psychology, University of Silesia, Katowice, Poland

4 Department of Oncology and the Department of Public Health Sciences, Queen's University, Kingston, ON, Canada meaningfully improve outcomes in pediatric cancers are needed [3, 4].

However, pediatric research on anticancer agents poses more challenges than research involving adult participants [5-7]. Children are considered a vulnerable population, thus their enhanced protection is required [8]. Despite challenges and strict requirements, conducting clinical trials in this population is indispensable to confirm the efficacy of tested drugs and to protect children against possible negative effects of prescribing medications off-label $[5,9,10]$.

Cancer phase II studies are an important element of the drug development process designed to establish the shortterm activity of new treatments, further evaluate safety and toxicity and, if these treatments are sufficiently promising, advance them for evaluation in phase III trials [11]. To be considered as ethical, research involving pediatric participants must meet, among others, a favorable risk/benefit ratio criterion [12-14]. The determination of whether risks and potential benefits of research interventions are acceptable requires ethical and clinical expertise and scientific knowledge and data [15]. Numerous studies have considered 


\section{Key Points}

We conducted a systematic review of activity and safety profiles of phase II targeted therapy clinical trials in pediatric oncology.

The pooled overall response rate was $24.4 \%$. The average drug-related grade $3 / 4$ adverse event rate per person was 0.66 . The overall drug-related fatal (grade 5) adverse event rate was $1.6 \%$.

These estimates of risks and benefits are helpful to make an informed decision about participation in phase II pediatric oncology trials testing targeted therapies.

adverse events (AEs) and response rates of anticancer agents in phase I adults as well as pediatric clinical trials [16-20]; others focused on the risks and benefits of particular drugs $[21,22]$ or interventions in specific cancer types [23, 24]. Historically, targeted drugs are considered to have a better toxicity profile than chemotherapy agents $[25,26]$. Yet, little is known about the risk-benefit profile for pediatric phase II oncology trials of targeted drugs. We conducted a systematic review and meta-analysis to systematically assess the risks and benefits for pediatric participants in contemporary phase II oncology clinical trials testing targeted therapies.

\section{Methods}

The study protocol was prospectively registered in PROSPERO (CRD42020146491) [27]. We followed the Preferred Reporting Items for Systematic Reviews and Meta-analyses guidelines.

\subsection{Search Strategy}

We systematically searched PubMed and EMBASE for relevant articles and abstracts published between 1 January, 2015 and 27 February, 2020, using strategies that included keywords and suggested MeSH and Emtree entry terms, their synonyms, and closely related words (Table 1 of the Electronic Supplementary Material [ESM]). Searches were not limited by language. Our search strategies were checked using the Canadian Agency for Drugs and Technologies in Health peer-review checklist for search strategies [28].

We performed a complementary search of ClinicalTrials. gov on 23 June, 2020 to find pediatric oncology studies with posted results (LZ, MTW). Then, all studies with registration numbers were searched by two researchers independently
(KS, MTW) for additional publications on ClinicalTrials. gov and/or Google Scholar on 3 July, 2020. For a detailed description of the search methodology, see the ESM.

\subsection{Study Selection and Eligibility Criteria}

Five researchers (LZ, MTW, KS, ES, MB) performed title and abstract screening for initial study inclusion and four researchers (LZ, MTW, ES, JS) performed full-text screening to determine the final list of included studies. The study selection process was conducted independently by two researchers, any disagreements were resolved by discussion, and when necessary an arbiter was involved (MW, BG).

We included pediatric cancer phase II clinical trials that published their results in the last 5 years as well as phase I/II or phase II/III studies (if phase II results were reported separately) investigating targeted therapy agents. Key inclusion criteria were as follows: (1) all or a majority of participants (over 50\%) were under 21 years old and/or the study was indicated as pediatric, or results were provided separately for the pediatric population; (2) diagnosis of any malignancy (solid or hematological); and (3) assessment of toxicity and/ or response of targeted therapy or a combination of targeted therapies (monoclonal antibodies or small molecules or antibody drug conjugates [29]). We excluded studies evaluating: (1) chemotherapy and immunotherapy regimens, or a combination of these with targeted therapy; (2) topical only or regionally administered drugs (i.e., delivered directly to the tumor without any systemic effects); (3) non-pharmacological modalities (e.g., surgery, radiotherapy, gene therapy, stem cell therapy, or any of these combined with targeted therapy); or (4) supportive care without anticancer agents or with other interventions not falling under a targeted therapy category (e.g., antiviral agents). Inclusion and exclusion criteria were defined prospectively in the protocol [27].

\subsection{Data Extraction}

We created and piloted a data extraction form. On the basis of the pilot, we refined and prepared the final version (available from https://osf.io/m7fw3/). Data were extracted from each publication and/or ClinicalTrials.gov study record independently by two reviewers (LZ, MTW, ES, JS). All reviewers received training prior to extraction. Disagreements were resolved by discussion, and when necessary, a third person, an arbiter, was involved (MW). An experienced medical oncologist had a supervisory role (BG). In the case of multiple publications for the same study, the results from the full publication and/or the most recent version were used in the extraction.

From each study, we extracted data related to: study characteristics (e.g., year of results publication, phase, funding, study status), patient characteristics (e.g., age, number of 
enrolled and eligible participants, type of malignancy), intervention (e.g., number and names of investigated drugs), and outcomes (e.g., toxicity, response). For more details, see our extraction form (https://osf.io/m7fw3/).

\subsection{Data Curation}

Phase II studies do not generally measure survival endpoints; thus, we decided to use the objective response rate as a proxy for the therapeutic benefit [30]. For solid tumor studies, we defined benefit as the proportion of participants with partial and/or complete response (reported separately or as an objective response rate) as defined by study authors. To measure benefit for hematological malignancy studies (except for acute leukemias and chronic myeloid leukemia), we considered any of the various methods of measuring partial and/or complete response (e.g., cytogenetic, hematologic, or molecular). For acute leukemias and chronic myeloid leukemia, we did not count any types of partial responses in our assessment of benefit because anything short of a complete response is not considered a benefit for these malignancies [31, 32].

We defined risk as the proportion of participants experiencing grade 3, 4, or 5 drug-related AEs as defined by the Common Toxicity Criteria for Adverse Events, Version 5.0 (and earlier versions) [33]. An AE was considered as related to the study drug if it was clearly stated by the study authors; expressions such as "AEs at least possibly related to study therapy", "treatment-emergent AEs", and "AEs suspected to be drug related" were also acceptable. In cases where a fatal event was not clearly described as treatment related, we excluded it from our drug-related grade $5 \mathrm{AE}$ rate estimations. All data on AEs in our analysis are treatment related. To compare the risks and benefits, we analyzed a cohort of studies where both drug-related deaths (grade 5 AEs) and responses were clearly reported.

\subsection{Statistical Analysis}

Pooled response rate, treatment-related fatal (grade 5) AE rate, and treatment-related grade 3/4 AEs rate were calculated within each stratum when more than one study provided data using meta-analytic methods. Modeling with random effects and the restricted maximum likelihood estimator were used to account for between-study heterogeneity. $I^{2}$ statistics were calculated to provide a measure of the proportion of overall variation attributable to between-study heterogeneity. Differences in response rates between the categories of study definition, number of drugs, and the number of types of malignancies were assessed using the $Q$ test for heterogeneity in meta-regression. Pooled response was calculated for categories of publication year (2015-16, 2017-18, and 2019-20) to assess changes over time. $P$-values for trends in response between 2015 and 2020 were obtained from a meta-regression. A meta-analysis was conducted using the metafor package (R, Version 3.2.3); $p<0.05$ was considered statistically significant. All tests were two-sided. The average number of treatment-related grade 3 and 4 AEs per person with a 95\% confidence interval (CI) was estimated using a Poisson regression model.

\subsection{Analysis of Both Solid Tumor and Hematological Malignancy Studies}

Because large differences were observed in responses between solid tumors and hematological malignancies, we stratified our analyses by the type of cancer. For three studies that included both solid and hematological malignancies, patients were separated for response data and, for one study including both these cancer types, we separated data on the drug-related fatal (grade 5) AE rate. The data separation did not influence response rates or drug-related fatal (grade 5) AEs rates in our analysis.

\subsection{Risk of Bias Assessment}

Two authors (LZ, MTW) independently assessed the risk of bias for all included studies using the Cochrane risk of bias tools for randomized or non-randomized studies [34, 35]. Judgments were based on the algorithms proposed by the authors of ROBINS and RoB2 tools, adjusted to fit the specific aspects of our analysis. Disagreements were resolved by discussion.

\section{Results}

\subsection{Clinical Trial and Patient Characteristics}

Our search identified 8317 references and 7375 remained after the removal of duplicates. We selected 119 full texts for review, of which 31 (concerning 25 clinical trials) met our inclusion criteria. After additional searches on ClinicalTrials.gov and Google Scholar, we included a total of 34 unique clinical trials for extraction (Fig. 1 of the ESM). The list of included studies with malignancy names and agents tested is presented in Table 2 of the ESM.

Among the 34 clinical trials $(N=1202)$ included, the majority $(28 ; 82.4 \%)$ were phase II trials, five $(14.7 \%)$ reported results of a phase I/II trial, and one (2.9\%) reported a phase II/III trial (Table 1). Twenty-seven trials $(79.4 \%)$ enrolled only pediatric patients, and seven (20.6\%) enrolled both children and adults. Twenty-two studies (64.7\%) involved only patients with solid tumors, while nine studies $(26.5 \%)$ involved only patients with hematological malignancies. The vast majority of trials $(31 ; 91.2 \%)$ tested 
monotherapies and three (8.8\%) tested combinations of two targeted agents. Twelve studies (35.4\%) recommended further drug evaluations, and three $(8.8 \%)$ were against further testing. Ten trials (29.4\%) were funded by private sponsors, $11(32.4 \%)$ by public institutions, and $12(35.3 \%)$ by both private and public sponsors. The majority of the trials (27; 79.4\%) were conducted in North America.

In 25 studies, the median age of participants was below 18 years, in three studies, the median age was between 18 and 21 years, and in the remaining six studies, the median age was not reported (Table 2). Twenty-one studies used the Lansky/Karnofsky performance scale, two studies used the Eastern Cooperative Oncology Group/World Health Organization/Zubrod scale, and seven studies used both these scales to measure performance status for eligibility.

\subsection{Benefit}

We analyzed 360 responses reported among 1202 participants enrolled in 34 clinical trials. The pooled overall response rate across all malignancies was $24.4 \%$ (95\% CI $14.5-34.2 ; I^{2}=98.5 \%$ ) (Table 3 ). The response rate for solid tumors among 670 patients was 6.4\% (95\% CI 3.2-9.6), while the response rate for hematological malignancies among 522 patients was significantly higher: $55.1 \%$ (95\% CI 35.9-74.3); $p<0.001$.

We did not find a significant difference in response rates by the type of trial (Table 3 of the ESM). The response rate in phase II/III trials vs phase I/II trials vs phase II trials was: $40.4 \%(N=1)$ vs $33.4 \%(N=6)$ vs $22.2 \%(N=30) ; p=$ 0.62 . The response rate in seamless phase I/II trials vs phase II trials in solid tumors was: $10.5 \%$ vs $3.6 \% ; p=0.08$.

We observed higher responses related to the smaller number of types of malignancies included in a study. The response rate was higher in all trials where three or fewer types of malignancies were treated $(30.3 \%)$ in comparison to the trials with four or more types of cancers $(2.3 \%) ; p=$ 0.047 .

The comparison of response rates between single-drug and multiple-drug studies was not possible because only three solid tumor studies investigated two drugs per study. In these three studies, none of the participants responded to the study drugs.

\subsection{Trends in Response Rate}

We did not find significant linear time trends in response rates ( $p=0.96$ for hematological malignancies, $p=0.27$ for solid tumors, $p=0.1$ for both hematological malignancies and solid tumors) (Fig. 1). The response rate for studies published between 2015 and 2016 was 9.2\% (95\% CI 3.2-15.1), and the response rate for trials published in 2017 or 2018 was $32.3 \%$ (95\% CI 16.4-48.2).

\subsection{Risk}

Twenty of the 34 trials reported drug-related fatal (grade 5) AEs. There were 16 drug-related grade 5 AEs among 694 patients, and the overall treatment-related grade $5 \mathrm{AE}$ rate was $1.6 \%\left(95 \%\right.$ CI $\left.0.6-2.5 \%, I^{2}=5.1 \%\right)$ (Table 3$)$. The difference in the drug-related grade $5 \mathrm{AE}$ rate between solid tumors and hematological malignancies was not statistically significant $(2.5 \%$ vs $0.8 \% ; p=0.1)$.

One hundred and forty-nine patients $(49.0 \%$; $95 \%$ CI 31.3-66.7) experienced treatment-related grade 3/4 AEs in 12 trials (Table 3 ). There was no significant difference in the occurrence of drug-related grade 3/4 AEs between patients with solid tumors $(49.2 \%)$ and patients with hematological malignancies $(48.3 \%) ; p=0.81$.

One hundred and thirty-one drug-related grade 3/4 AEs were reported in eight studies among 199 patients with solid tumors, with an average rate per person of 0.66 (95\% CI $0.55-0.78)$. There were no hematological studies reporting drug-related grade $3 / 4$ AEs.

\subsection{Direct Comparison of Risk and Benefit}

We identified 20 studies where both response rates and drugrelated deaths (grade 5 AEs) were clearly reported (Table 3 ). The response rate was significantly higher in hematological studies (36.0\%; 95\% CI 8.1-64.2) than in solid tumor studies $(2.2 \%$; 95\% CI $0.8-3.5) ; p<0.01$. As described above, the treatment-related grade $5 \mathrm{AE}$ rate was $0.8 \%$ in hematological studies and $2.5 \%$ in solid tumor trials. We found that the response rate was significantly lower in studies reporting drug-related grade 5 AEs than in the remaining studies lacking data on drug-related grade 5 AEs: $16.0 \%$ (95\% CI 6.0-26.0) vs 39.1\% (95\% CI 21.5-56.7); $p=0.009$.

\subsection{Risk of Bias Assessment}

The risk of bias of the included studies is available on https://osf.io/vcshp/. There was only one open-label randomized study with the overall risk of bias judgment of 'some concerns' mainly owing to some differences in the measurement of the outcome between two groups. Among 33 nonrandomized studies, two of them were assessed as having a critical risk of bias. The missing data were the major factor contributing to high levels of bias marked as "critical" or "serious".

\section{Discussion}

To our knowledge, we report the first systematic review of the risks and benefits of pediatric phase II cancer trials in the era of targeted therapy. Our findings suggest that, on 
Table 1 Characteristics of 34 included clinical trials

\begin{tabular}{|c|c|c|c|c|c|}
\hline \multirow[t]{2}{*}{ Characteristic } & \multirow[t]{2}{*}{ Category } & \multicolumn{3}{|c|}{ Type of tumor } & \multirow[t]{2}{*}{ Total } \\
\hline & & Solid & Hematological & Both & \\
\hline All studies & & $22(64.7)$ & $9(26.5)$ & $3(8.8)$ & $34(100)$ \\
\hline \multirow[t]{6}{*}{ Publication year } & 2015 & $7(31.8)$ & $1(11.1)$ & $0(0)$ & $8(23.5)$ \\
\hline & 2016 & $5(22.7)$ & $0(0)$ & $1(33.3)$ & $6(17.7)$ \\
\hline & 2017 & $2(9.1)$ & $3\left(33.4^{\mathrm{a}}\right)$ & $2(66.7)$ & $7(20.6)$ \\
\hline & 2018 & $5(22.7)$ & $4(44.4)$ & $0(0)$ & $9(26.5)$ \\
\hline & 2019 & $2(9.1)$ & $1(11.1)$ & $0(0)$ & $3(8.8)$ \\
\hline & 2020 & $1(4.6)$ & $0(0)$ & $0(0)$ & $1(2.9)$ \\
\hline \multirow[t]{7}{*}{ Locations $^{\mathrm{b}}$} & Africa & $0(0)$ & $1(11.1)$ & $0(0)$ & $1(2.9)$ \\
\hline & Asia & $0(0)$ & $3(33.3)$ & $0(0)$ & $3(8.8)$ \\
\hline & Australia & $4(18.2)$ & $3(33.3)$ & $0(0)$ & $7(20.6)$ \\
\hline & Europe & $8(36.4)$ & $5(55.6)$ & $1(33.3)$ & $14(41.2)$ \\
\hline & North America & $17(77.3)$ & 8 (88.9) & $2(66.7)$ & $27(79.4)$ \\
\hline & South America & $1(4.6)$ & $1(11.1)$ & $0(0)$ & $2(5.9)$ \\
\hline & Not reported & $1(4.6)$ & $0(0)$ & $0(0)$ & $1(2.9)$ \\
\hline \multirow[t]{4}{*}{ Funding } & Private & $6\left(27.2^{\mathrm{a}}\right)$ & $4(44.4)$ & $0(0)$ & $10(29.4)$ \\
\hline & Public & $10(45.5)$ & $1\left(11.2^{\mathrm{a}}\right)$ & $0(0)$ & $11(32.4)$ \\
\hline & Mixed & $5(22.7)$ & $4(44.4)$ & $3(100)$ & $12(35.3)$ \\
\hline & Unclear & $1(4.6)$ & $0(0)$ & $0(0)$ & $1(2.9)$ \\
\hline \multirow[t]{4}{*}{ Study status } & Active, not recruiting & $3(13.6)$ & $4(44.4)$ & $1(33.3)$ & $8(23.5)$ \\
\hline & Completed & $13(59.1)$ & $4(44.4)$ & $2(66.7)$ & $19(55.9)$ \\
\hline & Terminated & $4(18.2)$ & $1\left(11.2^{\mathrm{a}}\right)$ & $0(0)$ & $5(14.7)$ \\
\hline & Not reported & $2(9.1)$ & $0(0)$ & $0(0)$ & $2(5.9)$ \\
\hline \multirow[t]{3}{*}{ Study definition } & Phase I/II & $3(13.6)$ & $1(11.1)$ & $1(33.3)$ & $5(14.7)$ \\
\hline & Phase II & $19(86.4)$ & $7(77.8)$ & $2(66.7)$ & $28(82.4)$ \\
\hline & Phase II/III & $0(0)$ & $1(11.1)$ & $0(0)$ & $1(2.9)$ \\
\hline \multirow[t]{2}{*}{ Population } & Pediatric only & $16(72.7)$ & $8(88.9)$ & $3(100)$ & $27(79.4)$ \\
\hline & Mixed & $6(27.3)$ & $1(11.1)$ & $0(0)$ & $7(20.6)$ \\
\hline \multirow[t]{5}{*}{ Number of tumor types studied in the trial } & 1 type & $10(45.5)$ & $7(77.8)$ & $0(0)$ & $17(50.0)$ \\
\hline & 2 or 3 types & $5(22.7)$ & $2(22.2)$ & $1(33.3)$ & $8(23.5)$ \\
\hline & 4 types & $2(9.1)$ & $0(0)$ & $0(0)$ & $2(5.9)$ \\
\hline & More than 4 & $4\left(18.1^{\mathrm{a}}\right)$ & $0(0)$ & $2(66.7)$ & $6(17.7)$ \\
\hline & Not reported & $1(4.6)$ & $0(0)$ & $0(0)$ & $1(2.9)$ \\
\hline \multirow[t]{2}{*}{ Total number of investigational drugs } & 1 drug & $19(86.4)$ & $9(100)$ & $3(100)$ & $31(91.2)$ \\
\hline & 2 drugs & $3(13.6)$ & $0(0)$ & $0(0)$ & $3(8.8)$ \\
\hline \multirow[t]{3}{*}{ Randomization } & Yes & $1\left(4.5^{\mathrm{a}}\right)$ & $0(0)$ & $0(0)$ & $1(2.9)$ \\
\hline & No & $0(0)$ & $0(0)$ & $1(33.3)$ & $1(2.9)$ \\
\hline & Not applicable & $21(95.5)$ & $9(100)$ & $2(66.7)$ & $32\left(94.2^{\mathrm{a}}\right)$ \\
\hline \multirow[t]{2}{*}{ Blinding } & Blinding present & $0(0)$ & $0(0)$ & $0(0)$ & $0(0)$ \\
\hline & No blinding & $22(100)$ & $9(100)$ & $3(100)$ & $34(100)$ \\
\hline \multirow[t]{4}{*}{ Further studies recommended } & Yes & $7(31.8)$ & $3(33.3)$ & $2(66.7)$ & $12\left(35.4^{\mathrm{a}}\right)$ \\
\hline & No & $2(9.1)$ & $1(11.1)$ & $0(0)$ & $3(8.8)$ \\
\hline & Not reported & $12(54.6)$ & $5(55.6)$ & $1(33.3)$ & $18(52.9)$ \\
\hline & Not applicable & $1\left(4.5^{\mathrm{a}}\right)$ & $0(0)$ & $0(0)$ & $1(2.9)$ \\
\hline
\end{tabular}

Data given as number of studies (\%)

${ }^{a}$ Percentage was calculated by subtracting the remaining $\%$ values from $100 \%$

${ }^{\mathrm{b}}$ Some of the studies were conducted in more than 1 location 
Table 2 Characteristics of patients

\begin{tabular}{|c|c|c|c|c|c|}
\hline \multirow[t]{2}{*}{ Characteristic } & \multirow[t]{2}{*}{ Category } & \multicolumn{3}{|c|}{ Type of tumor } & \multirow[t]{2}{*}{ Total } \\
\hline & & Solid & Hematological & Both & \\
\hline \multicolumn{2}{|l|}{ Enrolled patients, $n$} & 559 & 491 & 152 & 1202 \\
\hline \multicolumn{2}{|l|}{ Evaluated patients, $n(\%)$} & $524(93.7)$ & $470(95.7)$ & $151(99.3)$ & $1145(95.3)$ \\
\hline \multicolumn{2}{|l|}{ Male, $n(\%)^{\mathrm{a}}$} & $290(51.9)$ & $214(43.6)$ & $80(52.6)$ & $584(48.6)$ \\
\hline \multirow{4}{*}{$\begin{array}{l}\text { Median age at enrollment, } n(\%) \\
\text { of studies }^{\mathrm{b}}, \text { years }\end{array}$} & $<6$ & $0(0)$ & $0(0)$ & $0(0)$ & $0(0)$ \\
\hline & $6.0-11.9$ & $9(40.9)$ & $3(33.3)$ & $2(66.7)$ & $14(41.2)$ \\
\hline & $12.0-17.9$ & $8(36.4)$ & $3(33.3)$ & $0(0)$ & $11(32.4)$ \\
\hline & $18-21$ & $2(9.1)$ & $1(11.1)$ & $0(0)$ & $3(8.8)$ \\
\hline \multirow{5}{*}{$\begin{array}{l}\text { Performance status scale used, } n \\
\quad(\%) \text { of studies }\end{array}$} & Lansky/Karnofsky scale & $16(72.7)$ & $4(44.4)$ & $1(33.3)$ & $21(61.8)$ \\
\hline & ECOG/WHO/Zubrod & $1(4.6)$ & $1\left(11.2^{\mathrm{d}}\right)$ & $0(0)$ & $2(5.9)$ \\
\hline & Both & $4\left(18.1^{\mathrm{d}}\right)$ & $2(22.2)$ & $1(33.3)$ & $7(20.6)$ \\
\hline & Other $^{\mathrm{c}}$ & $0(0)$ & $0(0)$ & $1\left(33.4^{\mathrm{d}}\right)$ & $1(2.9)$ \\
\hline & Not reported & $1(4.6)$ & $2(22.2)$ & $0(0)$ & $3(8.8)$ \\
\hline
\end{tabular}

ECOG Eastern Cooperative Oncology Group, WHO World Health Organization

${ }^{a}$ Sex was not reported in 1 mixed malignancy, 2 solid tumor, and 3 hematological malignancy studies

${ }^{\mathrm{b}}$ Age was not reported in 1 mixed malignancy, 3 solid tumor, and 2 hematological malignancy studies

'Other: Lansky/Karnofsky scale plus Standard Children's Oncology Group criteria for performance status

${ }^{\mathrm{d}}$ Percentage was calculated by subtracting the remaining \% values from $100 \%$

Table 3 Response rates and toxicity in the type of malignancy subgroups

\begin{tabular}{|c|c|c|c|c|}
\hline \multirow[t]{2}{*}{ Outcome } & \multirow[t]{2}{*}{ Measure } & \multicolumn{3}{|c|}{ Type of malignancy } \\
\hline & & Solid tumors & Hematological malignancies & Solid and hematological \\
\hline \multirow[t]{3}{*}{ Benefit (34 clinical trials ${ }^{\mathrm{a}}$ ) } & No. of studies & 25 & 12 & $34^{\mathrm{a}}$ \\
\hline & Response rate, $\%(95 \% \mathrm{CI})$ & $6.4(3.2-9.6)$ & $55.1(35.9-74.3)$ & $24.4(14.5-34.2)$ \\
\hline & $P$ value & $<0.001^{\mathrm{b}}$ & & - \\
\hline \multirow{3}{*}{$\begin{array}{l}\text { Drug-related fatal (grade 5) AEs } \\
\quad\left(20 \text { clinical trials }^{\mathrm{c}}\right)\end{array}$} & No. of studies & 15 & 6 & $20^{\mathrm{c}}$ \\
\hline & $\begin{array}{l}\text { Drug-related grade } 5 \mathrm{AE} \text { rate, } \% \\
\quad(95 \% \mathrm{CI})\end{array}$ & $2.5(1.1-4.0)$ & $0.8(0.01-1.8)$ & $1.6(0.6-2.5)$ \\
\hline & $P$ value & $0.1^{\mathrm{b}}$ & & - \\
\hline \multirow{3}{*}{$\begin{array}{l}\text { Benefit ( } 20 \text { clinical trials where } \\
\text { drug-related grade } 5 \text { AEs } \\
\text { reported }^{c} \text { ) }\end{array}$} & No. of studies & 15 & 6 & $20^{\mathrm{c}}$ \\
\hline & Response rate, $\%(95 \% \mathrm{CI})$ & $2.2(0.8-3.5)$ & $36.0(8.1-64.2)$ & $16.0(6.0-26.0)$ \\
\hline & $P$ value & $0.0018^{\mathrm{b}}$ & & - \\
\hline \multirow{3}{*}{$\begin{array}{l}\text { Patients with drug-related grade } \\
\text { 3/4 AEs (12 clinical trials) }\end{array}$} & No. of studies & 9 & 3 & 12 \\
\hline & $\begin{array}{l}\% \text { of patients with drug-related } \\
3 / 4 \text { AEs }(95 \% \text { CI })\end{array}$ & $49.2(29.0-68.4)$ & $48.3(6.6-93.6)$ & $49.0(31.3-66.7)$ \\
\hline & $P$ value & $0.81^{\mathrm{b}}$ & & - \\
\hline
\end{tabular}

$A E$ adverse event, $C I$ confidence interval

${ }^{a}$ Responses of 3 studies including both types of malignancies were analyzed separately

${ }^{\mathrm{b}} P$ value from $Q$ test for heterogeneity comparing response rates, drug-related grade 3/4 AEs rates, and drug-related grade 5 AE rates between types of malignancy

${ }^{c}$ Fatal (grade 5) AEs related to treatment of 1 study including both types of malignancies were analyzed separately

average, one in four children who enroll in pediatric phase II trials respond to a targeted therapy agent, while one in 63 die from drug-related AEs. However, the response rates and drug-related deaths vary from study to study and depend on the type of malignancy. For example, in our sample of 34 clinical trials, one study (NCT01460160) reported that 99 patients out of 109 patients enrolled (90.8\%) achieved complete remission; and another study (NCT03257631) reported 
Fig. 1 Time trends in response rates by type of malignancy. Response rates were calculated for categories of publication year (2015-16, 2017-18, and 2019-20). Error bars indicate standard error

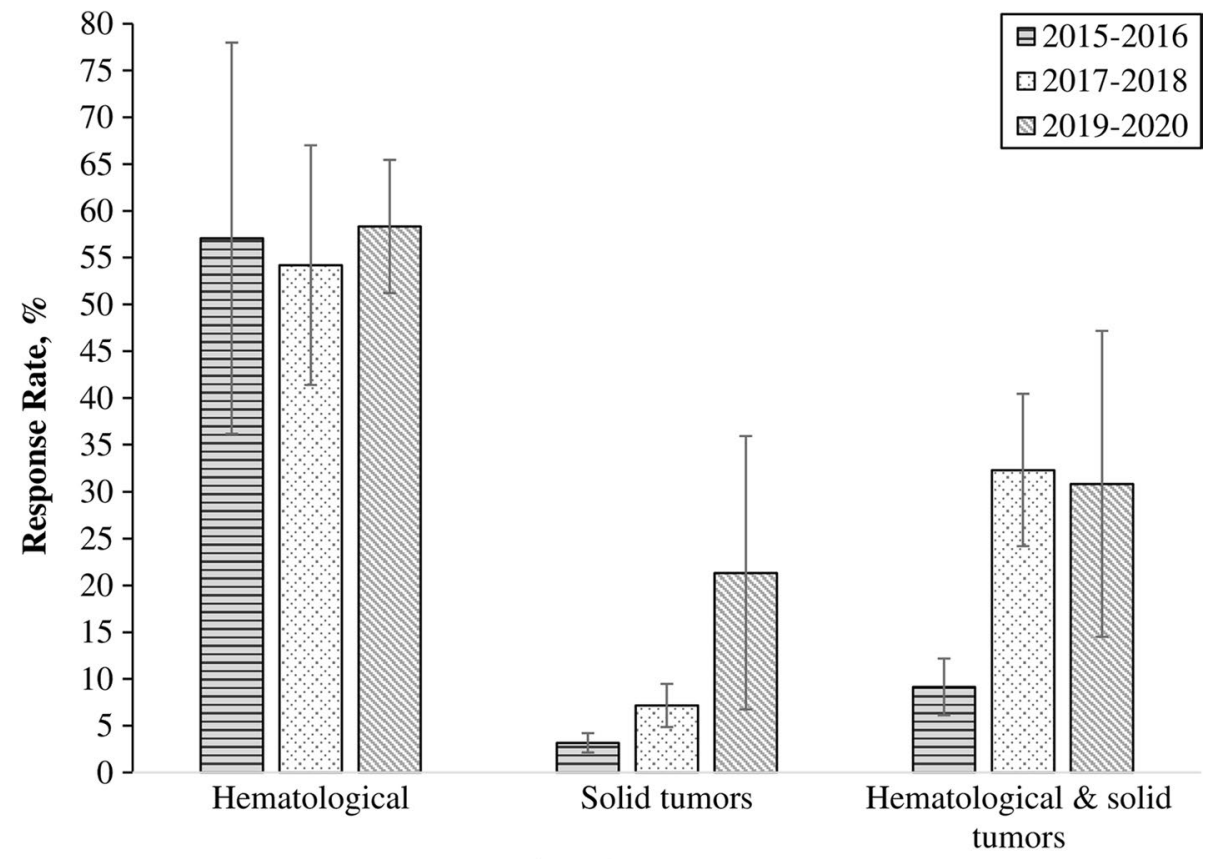

Type of malignancy ten treatment-emergent adverse events leading to death on 53 patients enrolled (18.9\%).

In our previous meta-analysis of 74 pediatric oncology, phase I targeted therapy studies enrolling 2264 patients, we reported that the pooled objective response rate for targeted therapy trials was: $3.5 \%$ (95\% CI 2.8-4.3), while the overall drug-related fatal (grade 5) AE rate was $1.8 \%(95 \% \mathrm{CI}$ 1.0-2.6) [17]. The overall response rate for solid tumor trials was lower in targeted phase I trials $(2.5 \%)$ than in phase II trials $(6.4 \%)$. This rate was also lower in hematological studies: $22.8 \%$ vs $55.1 \%$. In the case of a treatment-related grade $5 \mathrm{AE}$ rate for solid tumors, it was $1.7 \%$ in phase I trials vs $2.5 \%$ in phase II trials and for hematological malignancies: $3.2 \%$ vs $0.8 \%$. These findings support an expectation that pediatric phase II trials in oncology may pose a lower risk and a higher probability of direct benefit for the participants than phase I trials [17, 36].

Despite the growing role of targeted therapy, continuous innovation, and recent emphasis on precision oncology, we found that the overall response rate in phase II targeted therapy trials $(24.4 \%)$ is similar to the overall response rate in pediatric phase II cancer chemotherapy trials $(19.6 \% ; N$ $=45)$. The overall fatal AE rate in targeted therapy trials $(1.6 \%)$ was also similar to that reported with chemotherapy trials (1.4\%) [37]. However, a larger study of 570 phase II single-agent studies including 32,149 patients with nonpediatric cancers reports higher response rates for targeted therapy trials that adopted a personalized approach vs those that lacked a personalized strategy: median response $31.3 \%$ vs $7.5 \% ; p<0.0001$ [38]. The median treatment-related death rate for the personalized therapy approach was similar to our result $(1.5 \%)$ and slightly higher for all arms testing targeted agents (1.9\%) [38].

Similarly to our previous study [17], we found a significantly higher overall response rate in hematological malignancies compared with solid tumors. The reasons are biological differences between these malignancies and different criteria of response measurement. We also found that the studies including three or fewer types of malignancies have a higher probability of benefit than the studies with four or more malignancies. The systematic analysis by Franshaw et al. published in 2019 supports this claim [39].

We did not compare the response rates between singledrug and multiple-drug studies because the majority of trials in our sample were single-drug studies. Previous studies have shown that using a combination of a few agents increases the likelihood of benefiting in a trial [17, 39, 40].

We did not observe higher response rates in solid tumor seamless clinical trials than in traditional phase II clinical trials. The reason may be that only a few seamless clinical trials ultimately met our inclusion criteria and were analyzed.

Our findings should be interpreted in light of the following limitations. First, we used broad inclusion criteria to analyze the overall response rate and drug-related adverse events in all available results of phase II trials by including not only published articles but also abstracts and summary results posted on the ClinicalTrials.gov registry. This resulted in differences in the quality of outcomes reporting, which remains poor even in highly ranked oncology journals [41], and an overall serious risk of bias in the majority of included trials. For instance, we excluded 14 trials (41\%) 
from the meta-analysis of the drug-related grade $5 \mathrm{AE}$ rate and 22 trials $(65 \%)$ from the meta-analysis of drug-related grade 3 and 4 AEs, mainly because these outcomes were not clearly reported as treatment related. As a result, our risk estimates may be either over-estimated or underestimated. Moreover, participants' age was not reported in six trials (18\%). Second, in 14 clinical trials, the number of drugrelated deaths was reported to be zero while ten out of the total 16 treatment-related deaths were reported in one study (NCT03257631), which significantly influenced the overall drug-related grade $5 \mathrm{AE}$ rate. Third, the trials included in our analysis were heterogeneous. Generally, we observed greater heterogeneity in the measurement of response rate than the drug-related fatal $\mathrm{AE}$ rate, and in hematological malignancy studies compared with solid tumor studies. We reduced it by performing subgroup analyses within cancer types (solid tumors and hematological malignancies) according to the number of drugs tested in a trial, study type, and the number of malignancies included in a study. Fourth, we did not explore whether cancer phase II clinical trials integrating biomarkers as eligibility criteria have higher response rates, as it was observed in single-agent targeted therapy, phase I studies [20]. However, this limitation may determine a possible direction for future research. Fifth, objective response and progression-free survival are considered only surrogates for clinical benefit and do not necessarily translate to patientcentered outcomes as overall survival [42-47]. In addition, progression-free survival or overall survival are either not or inconsistently reported in phase II trials. For example, in our sample, three out of 34 studies reported median progressionfree survival, and one study reported median overall survival. Moreover, the reported survival rates included results for incomparable periods of time, with a range of 3 months to 10 years. Finally, all such analyses are necessarily fraught with publication bias as trials with poor response and significant toxicities may never be published while positive studies are rapidly published.

\section{Conclusions}

Our systematic review of phase II trials testing targeted therapy agents in pediatric oncology demonstrated risks and benefits, the acceptability of which must be judged by the physician and patient (and patient's guardians) on a caseby-case basis. Our data provide an empirical basis to current discussion regarding the risk/benefit profile in pediatric oncology research. It also contributes to communication about the benefits and burdens in pediatric phase II cancer trials to participants and their guardians.

Supplementary Information The online version contains supplementary material available at https://doi.org/10.1007/s11523-021-00822-5.
Acknowledgements The authors thank Phyllis Zych Budka for linguistic edits.

\section{Declarations}

Funding This work was supported by the National Science Center, Poland, UMO-2015/18/E/HS1/00354 (www.ncn.gov.pl). Mateusz T. Wasylewski, Karolina Strzebonska, Lucja Zaborowska, Maciej Polak, and Marcin Waligora (principal investigator) received the funding. The funders had no role in the study design, data collection and analysis, decision to publish, or preparation of the manuscript.

Conflict of interest Marcin Waligora reports personal fees from the Advisory Bioethics Council (Sanofi) outside the submitted work. Karolina Strzebonska, Mateusz T. Wasylewski, Lucja Zaborowska, Maciej Polak, Emilia Slugocka, Jakub Stras, Mateusz Blukacz, and Bishal Gyawali have no conflicts of interest that are directly relevant to the content of this article.

Ethics approval Not applicable.

Consent to participate Not applicable.

Consent for publication Not applicable.

Availability of data and material Data are available in the Open Science Framework online public database (https://osf.io/zhngb/). Additional data are available upon request.

Code availability Not applicable.

Author contributions MW, BG, and KS developed the concept for the research study. LZ, MTW, KS, ES, and MB performed abstract screening. LZ, MTW, ES, and JS conducted full-text screening and data extraction. LZ and MTW assessed the risk of bias. MP performed the statistical analyses. KS drafted the manuscript and was responsible for coordination of all aspects of the work. All the authors critically revised the manuscript for important intellectual content and approved the final version.

Open Access This article is licensed under a Creative Commons Attribution-NonCommercial 4.0 International License, which permits any non-commercial use, sharing, adaptation, distribution and reproduction in any medium or format, as long as you give appropriate credit to the original author(s) and the source, provide a link to the Creative Commons licence, and indicate if changes were made. The images or other third party material in this article are included in the article's Creative Commons licence, unless indicated otherwise in a credit line to the material. If material is not included in the article's Creative Commons licence and your intended use is not permitted by statutory regulation or exceeds the permitted use, you will need to obtain permission directly from the copyright holder. To view a copy of this licence, visit http://creativecommons.org/licenses/by-nc/4.0/.

\section{References}

1. Adamson P, Arons D, Baumberger J, Fleury M, Hoffman RI, Leach D, et al. Translating discovery into cures for children with cancer. Childhood cancer research landscape report. Alliance for Childhood Cancer and American Cancer Society; 2016. pp. 1-88. https://www.cancer.org/content/dam/cancer-org/research/ 
translating-discovery-into-cures-for-children-with-cancer-lands cape-report.pdf. Accessed 31 Mar 2021.

2. Atun R, Bhakta N, Denburg A, Frazier AL, Friedrich P, Gupta S, et al. Sustainable care for children with cancer: a Lancet Oncology Commission. Lancet Oncol. 2020;21(4):e185-224. https://doi.org/ 10.1016/S1470-2045(20)30022-X.

3. Wasylewski MT, Strzebonska K, Koperny M, Polak M, Kimmelman J, Waligora M. Clinical development success rates and social value of pediatric phase 1 trials in oncology. PLoS ONE. 2020;15(6):e0234911. https://doi.org/10.1371/journal.pone.02349 11.

4. Bedard PL, Hyman DM, Davis MS, Siu LL. Small molecules, big impact: 20 years of targeted therapy in oncology. Lancet. 2020;395(10229):1078-88. https://doi.org/10.1016/S01406736(20)30164-1.

5. Caldwell PHY, Murphy SB, Butow PN, Craig JC. Clinical trials in children. Lancet. 2004;364(9436):803-11. https://doi.org/10. 1016/S0140-6736(04)16942-0.

6. Podany AT. Ethical considerations in pediatric research. In: Buck ML, Manasco KB, editors. PedSAP 2017 book 1 research and study design in pediatrics. Lenexa: American College of Clinical Pharmacy; 2015.

7. Anderson B, Wolfe I, Muruganand A, Mody R. Accrual trends for Children's Oncology Group clinical trials: a single center experience. J Pediatr Hematol Oncol. 2020;42(7):e546-50. https://doi. org/10.1097/MPH.0000000000001938.

8. Wendler D. When and how to include vulnerable subjects in clinical trials. Clin Trials. 2020;17(6):696-702. https://doi.org/ 10.1177/1740774520945601.

9. Pandolfini C, Bonati M. A literature review on off-label drug use in children. Eur J Pediatr. 2005;164(9):552-8. https://doi.org/10. 1007/s00431-005-1698-8.

10. Lim M, Shulman DS, Roberts H, Li A, Clymer J, Bona K, et al. Off-label prescribing of targeted anticancer therapy at a large pediatric cancer center. Cancer Med. 2020;9(18):6658-66. https://doi. org/10.1002/cam4.3349.

11. Brown SR, Gregory WM, Twelves CJ, Brown J. A practical guide to designing phase II trials in oncology. New York: Wiley; 2014. https://doi.org/10.1002/9781118763612.

12. Wendler D. Three steps to protecting pediatric research participants from excessive risks. PLoS Clin Trial. 2006;1(5):e25. https://doi.org/10.1371/journal.pctr.0010025.

13. Waligora M, Strzebonska K, Wasylewski MT. Neither the harm principle nor the best interest standard should be applied to pediatric research. Am J Bioeth. 2018;18(8):72-4. https://doi.org/10. 1080/15265161.2018.1485762.

14. Kimmelman J, Waligora M, Lynch HF. Participant protection in phase 1 pediatric cancer trials. JAMA Pediatr. 2019;173(1):8-9. https://doi.org/10.1001/jamapediatrics.2018.3629.

15. Rid A, Wendler D. A framework for risk-benefit evaluations in biomedical research. Kennedy Inst Ethics J. 2011;21(2):141-79. https://doi.org/10.1353/ken.2011.0007.

16. Dorris K, Liu C, Li D, Hummel TR, Wang X, Perentesis J, et al. A comparison of safety and efficacy of cytotoxic versus molecularly targeted drugs in pediatric phase I solid tumor oncology trials. Pediatr Blood Cancer. 2017;64(3). https://doi.org/10.1002/pbc. 26258.

17. Waligora M, Bala MM, Koperny M, Wasylewski MT, Strzebonska $\mathrm{K}$, Jaeschke R, et al. Risk and surrogate benefit for pediatric phase I trials in oncology: a systematic review with meta-analysis. PLoS Med. 2018;15(2):e1002505. https://doi.org/10.1371/journal.pmed. 1002505 .

18. Cohen JW, Akshintala S, Kane E, Gnanapragasam H, Widemann BC, Steinberg SM. A systematic review of pediatric phase I trials in oncology: toxicity and outcomes in the era of targeted therapies.
Oncologist. 2020;25(6):532-40. https://doi.org/10.1634/theon cologist.2019-0615.

19. Hazim A, Mills G, Prasad V, Haslam A, Chen EY. Relationship between response and dose in published, contemporary phase I oncology trials. J Natl Compr Netw. 2020;18(4):428-33. https:// doi.org/10.6004/jncen.2019.7375.

20. Mackley MP, Fernandez NR, Fletcher B, Woolcott CG, Fernandez CV. Revisiting risk and benefit in early oncology trials in the era of precision medicine: a systematic review and meta-analysis of phase I trials of targeted single-agent anticancer therapies. JCO Precis Oncol. 2021;5:17-26. https://doi.org/10.1200/PO.20. 00214.

21. Carlisle B, Demko N, Freeman G, Hakala A, Mackinnon N, Ramsay T, et al. Benefit, risk, and outcomes in drug development: a systematic review of sunitinib. J Natl Cancer Inst. 2015;108(1):djv292. https://doi.org/10.1093/jnci/djv292.

22. Mattina J, Carlisle B, Hachem Y, Fergusson D, Kimmelman $\mathrm{J}$. Inefficiencies and patient burdens in the development of the targeted cancer drug sorafenib: a systematic review. PLoS Biol. 2017;15(2):e2000487. https://doi.org/10.1371/journal.pbio.20004 87.

23. Ljubas J, Ovesen T, Rusan M. A systematic review of phase II targeted therapy clinical trials in anaplastic thyroid cancer. Cancers. 2019;11(7):943. https://doi.org/10.3390/cancers11070943.

24. Silberholz J, Bertsimas D, Vahdat L. Clinical benefit, toxicity and cost of metastatic breast cancer therapies: systematic review and meta-analysis. Breast Cancer Res Treat. 2019;176(3):535-43. https://doi.org/10.1007/s10549-019-05208-w.

25. de Oliveira RL, Deschoemaeker S, Henze AT, Debackere K, Finisguerra V, Takeda Y, et al. Gene-targeting of Phd2 improves tumor response to chemotherapy and prevents side-toxicity. Cancer Cell. 2012;22(2):263-77. https://doi.org/10.1016/j.ccr.2012. 06.028.

26. Wang H, Yu J, Lu X, He X. Nanoparticle systems reduce systemic toxicity in cancer treatment. Nanomedicine. 2016;11(2):103-6. https://doi.org/10.2217/nnm.15.166.

27. Waligora M, Strzebonska K, Wasylewski M, Polak M, Gyawali B. Risk and benefit for pediatric phase II targeted therapy trials in oncology: a systematic review with meta-analysis. CRD42020146491. PROSPERO; 2020. https://www.crd.york. ac.uk/prospero/display_record.php?ID=CRD42020146491. Accessed 31 Mar 2021.

28. Canadian Agency for Drugs and Technologies in Health. CADTH peer review checklist for search strategies. Ottawa: The Agency; 2013. https://www.cadth.ca/sites/default/files/is/Peer_review/ CADTH\%20Peer\%20Review\%20Checklist\%20for\%20Search\% 20Strategies_e.pdf. Accessed 31 Mar 2021.

29. Karp DD, Falchook GS, editors. Handbook of targeted cancer therapy and immunotherapy. Targeted cancer therapy. 2nd ed. Philadelphia: Wolters Kluwer; 2018.

30. Dhani N, Tu D, Sargent DJ, Seymour L, Moore MJ. Alternate endpoints for screening phase II studies. Clin Cancer Res. 2009;15(6):1873-82. https://doi.org/10.1158/1078-0432. CCR-08-2034.

31. Center for International Blood and Marrow Transplant Research. AML response criteria. https://www.cibmtr.org/manuals/fim/1/en/ topic/aml-response-criteria. Accessed 6 May 2021.

32. Center for International Blood and Marrow Transplant Research. ALL response criteria. https://www.cibmtr.org/manuals/fim/1/en/ topic/all-response-criteria. Accessed 6 May 2021.

33. National Cancer Institute. Common terminology criteria for adverse events: (CTCAE). https://ctep.cancer.gov/protocoldevelop ment/electronic_applications/ctc.htm. Accessed 31 Mar 2021.

34. Sterne JAC, Hernán MA, Reeves BC, Savović J, Berkman ND, Viswanathan M, et al. ROBINS-I: a tool for assessing risk of bias 
in non-randomized studies of interventions. BMJ. 2016;355:i4919. https://doi.org/10.1136/bmj.i4919.

35. Sterne JAC, Savović J, Page MJ, Elbers RG, Blencowe NS, Boutron I, et al. RoB 2: a revised tool for assessing risk of bias in randomized trials. BMJ. 2019;366:14898. https://doi.org/10.1136/ bmj.14898.

36. Bautista F, Fioravantti V, de Rojas T, Carceller F, Madero L, Lassaletta A, et al. Medulloblastoma in children and adolescents: a systematic review of contemporary phase I and II clinical trials and biology update. Cancer Med. 2017;6(1):2606-24. https://doi. org/10.1002/cam4.1171.

37. Weitman S, Ochoa S, Sullivan J, Shuster J, Winick N, Pratt C, et al. Pediatric phase II cancer chemotherapy trials: a Pediatric Oncology Group study. J Pediatr Hematol Oncol. 1997;19(3):18791. https://doi.org/10.1097/00043426-199705000-00002.

38. Schwaederle M, Zhao M, Lee JJ, Eggermont AM, Schilsky RL, Mendelsohn J, et al. Impact of precision medicine in diverse cancers: a meta-analysis of phase II clinical trials. J Clin Oncol. 2015;33(32):3817-25. https://doi.org/10.1200/JCO.2015.61.5997.

39. Franshaw L, Tsoli M, Byrne J, Mayoh C, Sivarajasingam S, Norris $\mathrm{M}$, et al. Predictors of success of phase II pediatric oncology clinical trials. Oncologist. 2019;24(8):e765-74. https://doi.org/ 10.1634/theoncologist.2017-0666.

40. Azam F, Vazquez A. Trends in phase II trials for cancer therapies. Cancers (Basel). 2021;13(2):178. https://doi.org/10.3390/cance rs13020178.

41. Grellety T, Petit-Monéger A, Diallo A, Mathoulin-Pelissier S, Italiano A. Quality of reporting of phase II trials: a focus on highly ranked oncology journals. Ann Oncol. 2014;25(2):536-41. https:// doi.org/10.1093/annonc/mdt550.

42. Strzebonska K, Waligora M. Umbrella and basket trials in oncology: ethical challenges. BMC Med Ethics. 2019;20(1):58. https:// doi.org/10.1186/s12910-019-0395-5.

43. Gyawali B, D’Andrea E, Franklin JM, Kesselheim AS. Response rates and durations of response for biomarker-based cancer drugs in nonrandomized versus randomized trials. J Natl Compr Canc Netw. 2020;18(1):36-43. https://doi.org/10.6004/jncen.2019. 7345.

44. Gyawali B, Hey SP, Kesselheim AS. Evaluating the evidence behind the surrogate measures included in the FDA's table of surrogate endpoints as supporting approval of cancer drugs. EClinicalMedicine. 2020;21:100332. https://doi.org/10.1016/j.eclinm. 2020.100332.

45. Hwang TJ, Gyawali B. Association between progression-free survival and patients' quality of life in cancer clinical trials. Int J Cancer. 2019;144(7):1746-51. https://doi.org/10.1002/ijc.31957.

46. Gyawali B, Hey SP, Kesselheim AS. Assessment of the clinical benefit of cancer drugs receiving accelerated approval. JAMA Intern Med. 2019;179(7):906-13. https://doi.org/10.1001/jamai nternmed.2019.0462.

47. Kovic B, Jin X, Kennedy SA, Hylands M, Pedziwiatr M, Kuriyama A, et al. Evaluating progression-free survival as a surrogate outcome for health-related quality of life in oncology: a systematic review and quantitative analysis. JAMA Intern Med. 2018;178(12):1586-96. https://doi.org/10.1001/jamainternmed. 2018.4710 . 